\title{
AVALIAÇÃO DA CINÉTICA DE CRESCIMENTO DE PHORMIDIUM AUTUMNALE EM FOTOBIORREATORES
}

\author{
R. S. BIZELLO ${ }^{1}$, R. B. SARTORI ${ }^{1}$, L. G. RAMIREZ MERIDA ${ }^{1}$, L. Q. ZEPKA ${ }^{1}$, M. I. \\ QUEIROZ $^{2}$, E. JACOB-LOPES ${ }^{1}$ \\ ${ }^{1}$ Universidade Federal de Santa Maria (UFSM), Departamento de Tecnologia e Ciência de \\ Alimentos, CEP 97105-900 - Santa Maria, RS, Brasil. \\ ${ }^{2}$ Universidade Federal do Rio Grande (FURG), Departamento de Química, CEP 96201-900 - \\ Rio Grande, RS, Brasil. \\ E-mail para contato: jacoblopes@pq.cnpq.br
}

RESUMO - O objetivo do trabalho foi avaliar o crescimento da cianobactéria Phormidium autumnale em cultivo autotrófico com diferentes intensidades de iluminação em um fotobiorreator. Utilizou-se um fotobiorreator de coluna de bolhas com aeração constante de 1 VVM (volume de ar por volume de meio por minuto), concentração inicial de $\mathrm{CO}_{2}$ de $15 \%$, $100 \mathrm{mg} / \mathrm{L}$ de inóculo, temperatura constante de $25 \mathrm{C}^{\mathrm{o}}$ e intensidade luminosa numa faixa de 8000 a 15500 lux. Os resultados indicam que a produtividade máxima de biomassa foi de $21,21 \mathrm{mg} / \mathrm{L} . \mathrm{h}$, a uma taxa de fixação de carbono de $38,89 \mathrm{mg} / \mathrm{L} . \mathrm{h}$, em intensidade luminosa de 15500 lux.

\section{INTRODUÇÃO}

As microalgas vêm sendo utilizadas em processos biotecnológicos para a transformação e obtenção de produtos de interesse comercial, como por exemplo, biomassa, óleo e proteínas unicelulares, carboidratos, sais inorgânicos e compostos orgânicos voláteis, que são importantes nas indústrias de alimentos, química e farmacêutica (Queiroz et al., 2013). As microalgas são organismos autotróficos que apresentam organização celular procariótica e ausência de flagelo e da maioria das organelas celulares.

As cianobactérias constituem um grupo bem definido de eubactérias, sendo as únicas microalgas capazes de produzir oxigênio como produto colateral da fotossíntese. Clorofila $a$ e diversos pigmentos acessórios de proteção e ampliação da captação de luz são elementos que se fazem presente nas cianobactérias. (Kühl et al., 2005).

Phormidium autumnale é uma cianobactéria filamentosa de 3 a $4 \mu \mathrm{m}$ de diâmetro. Sua espécie é conhecida por viver em ambientes extremos, como fontes termais, solos de desertos e lugares contaminados. Por esta razão, apresentam grande potencial para o uso em processos biológicos com poucas exigências nutricionais (Guiry e Guiry, 2014). A obtenção intensiva de produtos através do cultivo microalgal pode ser realizada por meio de um fotobiorreator, que apresenta vantagens sobre outros tipos de reatores, por possuir maiores taxas de crescimento, melhor aproveitamento de $\mathrm{CO}_{2}$ e menor índice de contaminação. O metabolismo fotossintético destes microrganismos converte dióxido de carbono em produtos de alto valor agregado (Ramírez et al., 2013). Em face disto, o objetivo deste trabalho foi avaliar o 

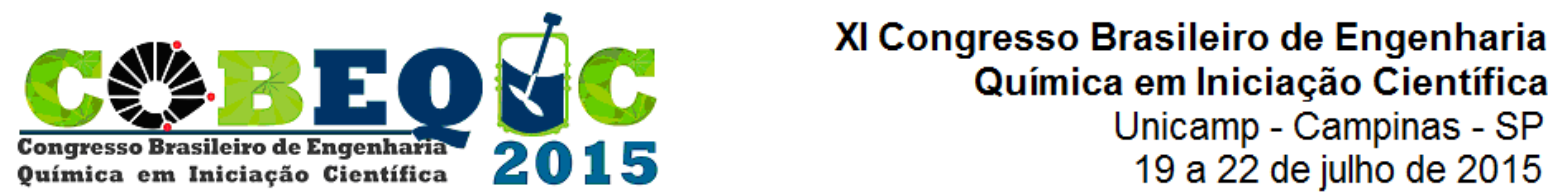

crescimento de Phormidium autumnale em cultivo autotrófico com diferentes intensidades de iluminação.

\section{MATERIAL E MÉTODOS}

A microalga utilizada foi a cianobactéria Phormidium autumnale obtida a partir de um isolamento de uma área localizada no deserto de Cuarto Cienégas $\left(26^{\circ} 59^{\prime} \mathrm{N}, 102^{\circ} 03^{\prime} \mathrm{W}-\right.$ México). A cultura estoque foi mantida e propagada em agar inclinado (20g/L) contendo meio sintético BG11 (Rippka et al., 1979) com a seguinte composição (g/L): $\mathrm{K}_{2} \mathrm{HPO}_{4}(0,03)$, $\mathrm{MgSO}_{4}(0,075), \mathrm{CaCl}_{2} \cdot 2 \mathrm{H}_{2} \mathrm{O}(0,036)$, citrato de amônio e ferro $(0,0006), \mathrm{Na}_{2} \operatorname{EDTA}(0,001)$, $\mathrm{NaCl}(0,00072), \mathrm{NaNO}_{3}(0,015)$, ácido cítrico $(0,0006), \mathrm{Na}_{2} \mathrm{CO}_{3}(1,5)$, traços de metais $\left[\mathrm{H}_{3} \mathrm{BO}_{3}(0,0028), \mathrm{MnCl}_{2} \cdot 4 \mathrm{H}_{2} \mathrm{O}(0,0018), \mathrm{ZnSO}_{4} \cdot 7 \mathrm{H}_{2} \mathrm{O}(0,00022), \mathrm{Na}_{2} \mathrm{MoO}_{4} \cdot 2 \mathrm{H}_{2} \mathrm{O}(0,00039)\right.$, $\left.\mathrm{CoSO}_{4} \cdot 6 \mathrm{H}_{2} \mathrm{O}(0,00004)\right] \mathrm{em}$ intensidade luminosa de $1 \mathrm{klux}, \mathrm{pH}$ de 7,6 , por um fotoperíodo de $12 \mathrm{~h}$.

Utilizou-se um fotobiorreator de coluna de bolhas operado com 2L de meio BG11 (Rippka et al, 1979), o qual foi suplementado com $15 \%$ de $\mathrm{CO}_{2}$ e aeração contínua de 1 VVM (volume de ar por meio de volume por minuto). As condições operacionais utilizadas foram: concentração celular inicial de $100 \mathrm{mg} / \mathrm{L}$, a temperatura constante de $25^{\circ} \mathrm{C}$ e intensidades luminosas no intervalo de 8000 a 15500 lux. Foram avaliadas a concentração celular e a taxa de fixação do carbono na biomassa, a cada $12 \mathrm{~h}$ por um máximo de $156 \mathrm{~h}$.

A concentração de biomassa celular foi determinada por gravimetria, usando filtros de $0,45 \mu \mathrm{m}$ de porosidade e $47 \mathrm{~mm}$ de diâmetro (Millex FG, Billerica, MA, EUA) (precisão de \pm $10 \%$ no método gravimétrico). A intensidade de luz foi medida com um luxímetro digital (MLM 1010, Minipa, São Paulo-SP, Brasil). A temperatura foi controlada através de um termostato ((HT-1300, ROXIN, China). Para a medição do fluxo de mistura de ar enriquecido com $\mathrm{CO}_{2}$ foram utilizados rotâmetros (KI-Key Instruments ${ }^{\circledR}$, Trevose, PA, EUA).

O tempo de geração, a velocidade máxima especifica de crescimento celular, a produtividade em biomassa e a taxa de remoção de carbono foram calculadas pelas equações 1-4.

$$
\begin{aligned}
& t_{g}(h)=\frac{\ln 2}{\mu} \\
& {\left[\ln \frac{x}{x_{0}}\right]\left(h^{-1}\right)=\mu_{\max } . t} \\
& \operatorname{Px}(m g / L . h)=\left(x_{i}-x_{i-1}\right)\left(t_{i}-t_{i-1}\right)
\end{aligned}
$$

$$
r_{c}(m g / L . h)=C b . P_{x}\left(\frac{M_{\mathrm{CO}_{2}}}{M_{C}}\right)
$$




\section{RESULTADOS E DISCUSSÃO}

A Tabela 1 apresenta os parâmetros cinéticos para Phormidium autumnale em função da intensidade luminosa. Obteve-se maior crescimento celular no tempo de residência de 108 $\mathrm{h}$ e intensidade luminosa de 15500 lux. Atingindo produtividade em biomassa de 21,21 $\mathrm{mg} / \mathrm{L} . \mathrm{h}$ com taxa de fixação de carbono de $38,89 \mathrm{mg} / \mathrm{L} . \mathrm{h}$. O tempo de geração $\left(\mathrm{t}_{\mathrm{g}}\right)$ foi de $34 \mathrm{~h}$ com uma máxima velocidade especifica de crescimento de $0,020 \mathrm{~h}^{-1}$. Esses resultados apresentaram-se 1,7 vezes abaixo do mostrado por Jacob Lopes et al. (2008) em cultivo de Aphanotece microscopica Nägeli utilizando a mesma concentração de $\mathrm{CO}_{2}$. No entanto são semelhantes aos valores reportados por Hsueh et al. (2007) e Sakai et al. (1995) para a microalga Chlorella sp.

Adicionalmente, verifica-se que com o aumento da intensidade luminosa há um aumento da produtividade de biomassa e velocidade de consumo de $\mathrm{CO}_{2}$ como fonte de carbono. Esses resultados estão de acordo com o apresentado por Liu et al. (2014) que avaliaram a fixação de carbono inorgânico dissolvido por Chlorella sp. e Scenedesmus obliquus, com cinco diferentes intensidades de iluminação em ciclos de 12/12h de luz/escuro, obtendo acima de $60 \%$ de remoção de carbono.

A partir da análise dos dados, observa-se uma variabilidade no desempenho do processo em função da intensidade luminosa, obtendo-se produtividades em biomassa entre 3,81 a $21,21 \mathrm{mg} / \mathrm{L}$.h e taxas de consumo de substrato entre 6,98 e 38,89mg/L.h. Evidenciando que as maiores taxas alcançadas foram em decorrência da intensidade luminosa de 15500 lux. Foi observada maior dependência em relação à intensidade de iluminação em comparação ao tempo de residência.

Tabela 1 - Parâmetros cinéticos para Phormidium em função da intensidade luminosa

\begin{tabular}{ccccccc}
\hline $\begin{array}{c}\text { Intensidade de } \\
\text { iluminação (lux) }\end{array}$ & $\begin{array}{c}\text { Tempo de } \\
\text { residência (h) }\end{array}$ & $\mathrm{X}_{\max }(\mathrm{mg} / \mathrm{L})$ & $\begin{array}{c}\mathrm{t}_{\mathrm{g}} \\
(\mathrm{h})\end{array}$ & $\begin{array}{c}\mu_{\max } \\
\left(\mathrm{h}^{-1}\right)\end{array}$ & $\begin{array}{c}\mathrm{P}_{\mathrm{X}} \max \\
(\mathrm{mg} / \mathrm{L} \cdot \mathrm{h})\end{array}$ & $\begin{array}{c}\mathrm{r}_{\mathrm{c}} \max \\
(\mathrm{mg} / \mathrm{L} . \mathrm{h})\end{array}$ \\
\hline 8000 & 96 & 380 & 40 & 0,017 & 3,81 & 6,98 \\
10500 & 84 & 580 & 38 & 0,018 & 10,56 & 19,35 \\
12000 & 96 & 380 & 21 & 0,019 & 11,55 & 21,18 \\
13000 & 108 & 700 & 37 & 0,019 & 13,02 & 23,87 \\
15500 & 108 & 1050 & 34 & 0,020 & 21,21 & 38,89 \\
\hline
\end{tabular}




\section{CONCLUSÃO}

As células expostas a intensidades luminosas adequadas apresentaram melhor desempenho quanto à produtividade de biomassa e consumo de carbono. Em intensidades luminosas de 15500 lux, evidenciou-se produtividades em biomassa de $21,21 \mathrm{mg} / \mathrm{L} . \mathrm{h}$ em paralelo a taxas de fixação de carbono de $38,89 \mathrm{mg} / \mathrm{L} . \mathrm{h}$.

\section{NOMENCLATURA}

$C b=$ Porcentagem de carbono celular $(\%)$

$M_{\mathrm{CO}_{2}}=$ Massa molecular de dióxido de carbono $(\mathrm{g} / \mathrm{mol})$

$M_{C}=$ Massa molecular de carbono $(\mathrm{g} / \mathrm{mol})$

$\mu=$ Velocidade máxima $\left(\mathrm{h}^{-1}\right)$

$\mu_{\max }=$ Velocidade máxima especifica de crescimento celular $\left(\mathrm{h}^{-1}\right)$

$\mathrm{P} x=$ Produtividade em biomassa (mg/L.h)

$r_{\mathrm{c}}=$ Taxa de remoção de carbono (mg/L.h)

$t=$ Tempo $(\mathrm{h})$

$t_{\mathrm{g}}=$ Tempo de geração $(\mathrm{h})$

$x=$ Concentração celular $(\mathrm{mg} / \mathrm{L})$

$x_{0}=$ Concentração celular inicial $(\mathrm{mg} / \mathrm{L})$

\section{REFERÊNCIAS}

GUIRY, M.D., GUIRY, G. M. AlgaeBase:World-wide electronic publication, National University of Ireland, Galway. from. http://www.algaebase.org, Retrieved 21 June 2014.

HSUEH H.T., CHU H., YU S.T. A batch study on the bio-fixation of carbon dioxide in the absorbed solution from a chemical wet scrubber by hot springs and marine algae, Chemosphere. 66 p.878-886, 2007.

JACOB-LOPES, E., LACERDA, L.M.C.F., FRANCO, T.T. Biomass production and carbon dioxide fixation by Aphanothece microscopica Nägeli in a bubble column photobioreactor. Biochem. Eng. J. v.40, p.27-34, 2008. 
KÜHL, M.; CHEN, M.; RALPH, P.J.; SCHREIBER, U.; LARKUM, A.W.D. A niche for cyanobacteria containing chlorophyll.d. Nature. v.433, p.820-820, 2005.

LIU G, QIAO L, ZHANG H, ZHAO D, SU X. The effects of illumination factors on the growth and $\mathrm{HCO} 3$ - fixation of microalgae in an experiment culture system. Energy, In Press, Corrected Proof, Available online 11 June 2014.

QUEIROZ, M.I, HORNES, M.O, MANETTI, A.G.S, ZEPKA, L.Q, JACOB-LOPES, E. Fish processing wastewater as a platform of the microalgae biorefineries. Biosystems Eng. p.195 e 202, Publicado on-line 20 de abril de 2013.

RAMÍREZ-MÉRIDA L., ZEPKA Q.L., JACOB-LOPES E. Fotobiorreactor: herramienta para cultivo de cianobacterias. Cienc. Tecnol. p.9-19, 2013.

RIPPKA, R.; DERUELLES, J.; WATERBURY, J.B.; HERDMAN, M.; STANIER, R.Y. Generic assignments strain histories and properties of pure cultures of cyanobacteria. J. Gen. Microbiol, v.111, p.1-61, 1979.

SAKAI N, SAKAMOTO Y, KISHIMOTO N, CHIHARA M, KARUBE I. Chlorella strains from hotsprings tolerant to high-temperature and high CO2. Energy Convers. Manage. V.36, p.693-696, June-September 1995. 